\title{
Numerical approximation of a concrete carbonation model: study of the $\sqrt{t}$-law of propagation
}

\author{
Antoine Zurek ${ }^{1}$
}

\begin{abstract}
In this paper, we are interested in the long time behavior of approximate solutions to a free boundary model which appears in the modeling of concrete carbonation [1]. In particular, we study the long time regime of the moving interface. The numerical solutions are obtained by an implicit in time and finite volume in space scheme. We show the existence of solutions to the scheme and, following $[2,3]$, we prove that the approximate free boundary increases in time following a $\sqrt{t}$-law. Finally, we supplement the study through numerical experiments.
\end{abstract}

\section{Introduction}

The carbonation phenomenon in reinforced concrete is a physico-chemical reaction which produces a moving interface inside the concrete. The carbonation process can be described as follows: $\mathrm{CO}_{2}$ in gaseous phase which comes from the atmosphere is quickly transformed in $\mathrm{CO}_{2}$ in aqueous phase. The transformation of $\mathrm{CO}_{2}(\mathrm{~g})$ to $\mathrm{CO}_{2}(\mathrm{aq})$ leads to the carbonation reaction when $\mathrm{CO}_{2}(\mathrm{aq})$ reacts with $\mathrm{Ca}(\mathrm{OH})_{2}(\mathrm{aq})$. This reaction produces a moving interface which splits the concrete in two parts: the carbonated one which grows in time and the uncarbonated one. We refer to $[1,2,3,6,8]$ for more details on the concrete carbonation reaction.

In [1], Aiki and Muntean have proposed a free boundary system in one dimension in space modeling concrete carbonation. This model consists in a system of two weakly coupled reaction-diffusion equations in a varying domain, the carbonated zone, whose length is governed by an ordinary differential equation. In this model, the unknowns $u$ and $v$ represent the mass concentration of $\mathrm{CO}_{2}$ respectively in aqueous and gaseous phase and $s$ represents the penetration depth which measures the size of the carbonated zone. Let us mention that this system is derived from [6]. In this paper, we consider the numerical approximation of the model proposed in [1]. As mentioned above, this system is defined on a varying domain. For numerical reasons, it is convenient to rewrite this model on a fixed domain. To this end, we use a change of variables [8]. Then, in the new coordinate system we consider

$$
Q(T)=\{(t, x): 0<x<1,0<t<T\} .
$$

\footnotetext{
${ }^{1}$ Univ. Lille, CNRS, UMR 8524, Inria - Laboratoire Paul Painlevé, F-59000 Lille; antoine.zurek@univ-lille.fr
} 
So that, we can rewrite the model in [1] as a convection-diffusion-reaction system defined by:

$$
\begin{gathered}
s(t) \partial_{t}(s(t) u)+\partial_{x} J_{u}=s^{2}(t) f(u, v) \quad \text { in } Q(T), \\
s(t) \partial_{t}(s(t) v)+\partial_{x} J_{v}=-s^{2}(t) f(u, v) \text { in } Q(T), \\
s^{\prime}(t)=\psi(u(1, t)) \text { for } 0<t<T, \\
u(0, t)=g(t) \quad \text { for } \quad 0<t<T, \\
v(0, t)=r(t) \quad \text { for } \quad 0<t<T, \\
J_{u}(1, t)=s(t) \psi(u(1, t)) \text { for } 0<t<T, \\
J_{v}(1, t)=0 \text { for } 0<t<T, \\
u(x, 0)=u_{0}\left(s_{0} x\right) \text { for } 0<x<1, \\
v(x, 0)=v_{0}\left(s_{0} x\right) \text { for } 0<x<1, \\
s(0)=s_{0} .
\end{gathered}
$$

The general convection-diffusion fluxes are defined by

$$
J_{w}=-\kappa_{w} \partial_{x} w-s(t) s^{\prime}(t) x w,
$$

where $w=u$ or $v$. We refer to [1], where existence and uniqueness of a global solution to (1) are established. As in the theoretical analysis, we suppose that the following assumptions are satisfied:

(A1) $\quad \psi: \mathbb{R} \longrightarrow \mathbb{R}$ represents the kinetics of the reaction and is defined by $\psi(x)=\alpha x^{p}$ with $\alpha>0$ and $p \geq 1$,

$(A 2) \quad f: \mathbb{R}^{2} \longrightarrow \mathbb{R}$ is given by the Henry's law and is defined by $f(u, v)=\beta(\gamma v-u)$ with $\beta$ and $\gamma$ two positive constants,

(A3) $g$ and $r$ belong to $H^{1}(0, T)$,

(A4) $u_{0}$ and $v_{0}$ belong to $L^{\infty}\left(\left[0, s_{0}\right]\right)$,

(A5) the diffusive coefficients $\kappa_{u}$ and $\kappa_{v}$ are two positive constants,

(A6) $s_{0}>0$,

(A7) there exist $g^{*}$ and $r^{*}$ two positive constants with $g^{*}=\gamma r^{*}$ such that

$$
\begin{aligned}
& 0 \leq g \leq g^{*} \text { and } 0 \leq r \leq r^{*} \quad \text { on } \quad[0,+\infty[, \\
& 0 \leq u_{0} \leq g^{*} \text { and } 0 \leq v_{0} \leq r^{*} \quad \text { on }\left[0, s_{0}\right] .
\end{aligned}
$$

In $[2,3]$, Aiki and Muntean show that the penetration depth $s$ follows a $\sqrt{t}$-law of propagation for constant Dirichlet boundary conditions. In this case, they prove the existence of two positive constants $c$ and $C$ independent of $t$ such that

$$
c \sqrt{t} \leq s(t) \leq C \sqrt{1+t}, \quad \forall t \geq 0 .
$$

They extend their result to the case of time dependent Dirichlet boundary conditions in [4]. We notice that there exists a wide literature in the continuous setting on the long time behavior of the free interface for Stefan like problem, see for instance $[9,10,11,14]$ and references therein. However, up to our knowledge, there exist no such results in the discrete setting.

In [8], we propose and show the convergence of a finite volume scheme for (1) and we observe that the approximate penetration depth follows a $\sqrt{t}$-law of propagation. The aim of this paper is to establish an inequality similar to (2). 
In $[2,3]$, the key idea is to prove an energy equality and then to deduce the $\sqrt{t}$-bounds satisfied by $s$. In the discrete setting the main difficulty is to define a scheme which permits to adapt this proof. To this end, we need to modify the scheme proposed in [8] and we will consider in this paper a fully implicit in time and finite volume in space scheme.

The paper is organized as follows: Section 2 introduces the numerical scheme and states the main results. Theorem 2.1 gives the existence of a solution to the scheme and Theorem 2.2 gives the long time behavior of the approximate penetration depth. Section 3 is devoted to the proof of Theorem 2.1, while we establish in Section 4 a discrete $L^{2}\left(0, T ; H^{1}(0,1)\right)$ estimate needed for the proof of Theorem 2.2. Theorem 2.2 is then proved in Section 5. We present some numerical results obtained with the scheme in Section 6. Finally, an Appendix gives a result required for the theoretical study of the scheme.

\section{Numerical scheme and main results}

\subsection{The fully implicit finite volume scheme}

In order to write a finite volume scheme we introduce notations related to the discretisation of $[0,1] \times[0, T]$. A mesh $\mathcal{T}$, consists in a finite sequence of cells denoted $\left(x_{i-\frac{1}{2}}, x_{i+\frac{1}{2}}\right)$, for $1 \leq i \leq l$, with

$$
0=x_{\frac{1}{2}}<x_{\frac{3}{2}}<\ldots<x_{l-\frac{1}{2}}<x_{l+\frac{1}{2}}=1 .
$$

We note $h_{i}=x_{i+\frac{1}{2}}-x_{i-\frac{1}{2}}$, for $1 \leq i \leq l$, the length of the $i$-th cell. The mesh size is defined as $h=\max \left\{h_{i}, 1 \leq i \leq l\right\}$. Moreover, for $1 \leq i \leq l$, we define $x_{i}$ as the center of the cell $\left(x_{i-\frac{1}{2}}, x_{i+\frac{1}{2}}\right), x_{0}=x_{\frac{1}{2}}$ and $x_{l+1}=x_{l+\frac{1}{2}}$. We set

$$
h_{i+\frac{1}{2}}=x_{i+1}-x_{i} \text { for } 0 \leq i \leq l .
$$

For the discretisation of $[0, T]$, we define a time step $\Delta t$ and an integer $N_{T}$ such that $N_{T} \Delta t=T$. We consider the sequence $\left(t_{n}\right)_{0 \leq n \leq N_{T}}$ with $t_{n}=n \Delta t$.

Then, for $1 \leq i \leq l$ and $0 \leq n \leq N_{T}-1$, the scheme writes

$$
\begin{gathered}
s^{n+1}=s^{n}+\Delta t \psi\left(u_{l+1}^{n+1}\right), \\
h_{i} s^{n+1} \frac{s^{n+1} u_{i}^{n+1}-s^{n} u_{i}^{n}}{\Delta t}+\left(\mathcal{F}_{u, i+\frac{1}{2}}^{n+1}-\mathcal{F}_{u, i-\frac{1}{2}}^{n+1}\right)= \\
h_{i}\left(s^{n+1}\right)^{2} f\left(u_{i}^{n+1}, v_{i}^{n+1}\right), \\
h_{i} s^{n+1} \frac{s^{n+1} v_{i}^{n+1}-s^{n} v_{i}^{n}}{\Delta t}+\left(\mathcal{F}_{v, i+\frac{1}{2}}^{n+1}-\mathcal{F}_{v, i-\frac{1}{2}}^{n+1}\right)= \\
-h_{i}\left(s^{n+1}\right)^{2} f\left(u_{i}^{n+1}, v_{i}^{n+1}\right) .
\end{gathered}
$$

It remains to define the numerical fluxes. We define

$$
\sigma^{n+1}=s^{n+1} \frac{s^{n+1}-s^{n}}{\Delta t},
$$


we introduce the local numerical Péclet number

$$
P_{w, i+\frac{1}{2}}^{n+1}=\frac{h_{i+\frac{1}{2}} \sigma^{n+1} x_{i+\frac{1}{2}}}{\kappa_{w}}, \quad \forall 0 \leq i \leq l,
$$

and we define a generic numerical flux, that is:

$$
\mathcal{F}_{w, i+\frac{1}{2}}^{n+1}=\kappa_{w} \frac{B\left(P_{w, i+\frac{1}{2}}^{n+1}\right) w_{i}^{n+1}-B\left(-P_{w, i+\frac{1}{2}}^{n+1}\right) w_{i+1}^{n+1}}{h_{i+\frac{1}{2}}}, \quad \forall 0 \leq i \leq l,
$$

for $w=u$ or $v$. For $B$, we should consider the upwind fluxes $B(x)=B_{u p}(x)=$ $1+x^{-}$with $x^{-}=\max (-x, 0)$ (see [7]) or the Scharfetter-Gummel fluxes $B(x)=$ $B_{s g}(x)=x /\left(e^{x}-1\right)$ with $B(0)=1$ introduced by Il'in in [12] and Scharfetter and Gummel in [13]. We notice that in both cases the function $B$ satisfies the following assumptions:

$$
\begin{aligned}
& B \text { is } L_{B} \text {-Lipschitz continuous on } \mathbb{R}, \\
& B(0)=1 \text { and } B(x)>0, \quad \forall x \in \mathbb{R}, \\
& B(x) \leq 1, \quad \forall x \in \mathbb{R}^{+}, \\
& B(x)-B(-x)=-x, \quad \forall x \in \mathbb{R} .
\end{aligned}
$$

We supplement the numerical scheme with the discretization of the boundary conditions

$$
v_{0}^{n}=r^{n}=\frac{1}{\Delta t} \int_{t_{n}}^{t_{n+1}} r(t) d t, \quad u_{0}^{n}=g^{n}=\frac{1}{\Delta t} \int_{t_{n}}^{t_{n+1}} g(t) d t,
$$

for $0 \leq n \leq N_{T}$ and

$$
\begin{gathered}
\mathcal{F}_{v, l+\frac{1}{2}}^{n+1}=0, \\
\mathcal{F}_{u, l+\frac{1}{2}}^{n+1}=s^{n+1} \psi\left(u_{l+1}^{n+1}\right) .
\end{gathered}
$$

Moreover, thanks to (3) and (6), we have for $0 \leq n \leq N_{T}$

$$
\mathcal{F}_{u, l+\frac{1}{2}}^{n+1}=s^{n+1} \psi\left(u_{l+1}^{n+1}\right)=\sigma^{n+1} .
$$

Finally, for the initial conditions we define for $1 \leq i \leq l$

$$
\begin{gathered}
s^{0}=s_{0}, \\
w_{i}^{0}=\frac{1}{h} \int_{x_{i-\frac{1}{2}}}^{x_{i+\frac{1}{2}}} w_{0}\left(s_{0} x\right) d x \text { and } w_{l+1}^{0}=w_{0}\left(s_{0}\right),
\end{gathered}
$$

for $w=u$ or $v$. We denote by $(S)$ the scheme $(3)-(15)$.

Remark 2.1. Let us notice that thanks to the hypothesis $(9 d)$ we have for $1 \leq i \leq l$ and $n \geq 0$ two decomposition formulae for the fluxes, either an upwind reformulation

$$
\mathcal{F}_{w, i+\frac{1}{2}}^{n+1}=-\kappa_{w} B\left(P_{w, i+\frac{1}{2}}^{n+1}\right) \frac{w_{i+1}^{n+1}-w_{i}^{n+1}}{h_{i+\frac{1}{2}}}-\sigma^{n+1} x_{i+\frac{1}{2}} w_{i+1}^{n+1},
$$


or a centered one

$$
\begin{aligned}
& \mathcal{F}_{w, i+\frac{1}{2}}^{n+1}=-\kappa_{w} B^{c}\left(P_{w, i+\frac{1}{2}}^{n+1}\right) \frac{w_{i+1}^{n+1}-w_{i}^{n+1}}{h_{i+\frac{1}{2}}} \\
&-\sigma^{n+1} x_{i+\frac{1}{2}} \frac{w_{i}^{n+1}+w_{i+1}^{n+1}}{2},
\end{aligned}
$$

with

$$
B^{c}(x)=\frac{B(x)+B(-x)}{2}, \quad \forall x \in \mathbb{R}
$$

\subsection{Main results}

First, we state the existence of a solution to the scheme $(S)$. Let us introduce for a given $\mathcal{T}$ the compact and convex set

$$
\mathbb{K}=\left\{(u, v) \in \mathbb{R}^{l+2} \times \mathbb{R}^{l+2}: 0 \leq u_{i} \leq g^{*}, \quad 0 \leq v_{i} \leq r^{*}, \quad \forall 0 \leq i \leq l+1\right\} .
$$

Theorem 2.1. Under the assumptions $(A 1)-(A 7)$ and (9) and for a given mesh $\mathcal{T}$ and a given $\Delta t$ then $(S)$ admits a solution $\left(s^{n}, u^{n}, v^{n}\right)$ for all $0 \leq n \leq N_{T}$ such that

$$
\left(u^{n}, v^{n}\right) \in \mathbb{K} \quad \text { and } \quad s^{n} \geq s^{0}>0, \quad \forall 0 \leq n \leq N_{T} .
$$

Moreover

$$
0 \leq \frac{s^{n+1}-s^{n}}{\Delta t} \leq \alpha\left(g^{*}\right)^{p}, \quad \forall 0 \leq n \leq N_{T}-1 .
$$

We prove Theorem 2.1 in Section 3. The proof is based on the Brouwer's fixed-point theorem.

As already said, our main result concerns the long time behavior of the approximate penetration depth. This result is given in Theorem 2.2.

Theorem 2.2. For a given $\Delta t$, let the hypothesis $(A 1)-(A 7)$ and $(9)$ hold, assume that $g(t)=g^{*}$ and $r(t)=r^{*}$ for $t \in[0,+\infty)$ with $g^{*}+r^{*}<1$. Then, there exist two positive constants $c$ and $C$ independent of $\Delta t$ such that

$$
c \sqrt{T} \leq s^{N_{T}} \leq C \sqrt{T+1}
$$

We show Theorem 2.2 in Section 5. For the proof, we establish two energy inequalities, see Proposition 5.1 and Proposition 5.2. Then, we prove the lower and upper bound of (21).

\section{Existence of a solution to the scheme}

In this section, we prove Theorem 2.1. The proof of existence is done by induction on $n$ and we follow some ideas developed in [5]. Let us note that the element $s^{0}$ is defined by (14) and the vectors $u^{0}$ and $v^{0}$ are defined by (15). Hypothesis $(A 7)$ ensures that $u^{0}$ and $v^{0}$ fulfill the condition (19).

We suppose that, for some $n \geq 0,\left(s^{n}, u^{n}, v^{n}\right)$ is known and satisfies (19) 
and (20). We want to establish the existence of $\left(s^{n+1}, u^{n+1}, v^{n+1}\right)$ solution to $(S)$ satisfying (19) and (20). To this end, we introduce the application $T^{n}: \mathbb{K} \longrightarrow \mathbb{R}^{l+2} \times \mathbb{R}^{l+2}$, such that $T^{n}(u, v)=(\hat{u}, \hat{v})$. The definition of $T^{n}$ is based on the linear scheme proposed in [8] and defined in two steps.

- First, for $(u, v) \in \mathbb{K}$ we define the element

$$
\hat{s}=s^{n}+\Delta t \alpha\left(u_{l+1}\right)^{p} .
$$

- Then, we define $(\hat{u}, \hat{v})$ as the solution to the following linear scheme

$$
\begin{aligned}
& h_{i} \hat{s} \frac{\hat{s} \hat{v}_{i}-s^{n} v_{i}^{n}}{\Delta t}+\left(\hat{G}_{\hat{v}, i+\frac{1}{2}}-\hat{G}_{\hat{v}, i-\frac{1}{2}}\right)=-h_{i}(\hat{s})^{2} f\left(u_{i}, \hat{v}_{i}\right), \\
& h_{i} \hat{s} \frac{\hat{s} \hat{u}_{i}-s^{n} u_{i}^{n}}{\Delta t}+\left(\hat{G}_{\hat{u}, i+\frac{1}{2}}-\hat{G}_{\hat{u}, i-\frac{1}{2}}\right)=h_{i}(\hat{s})^{2} f\left(\hat{u}_{i}, \hat{v}_{i}\right),
\end{aligned}
$$

for $1 \leq i \leq l$ and

$$
\hat{G}_{\hat{v}, l+\frac{1}{2}}=0, \quad \hat{G}_{\hat{u}, l+\frac{1}{2}}=\hat{s} \alpha \hat{u}_{l+1}\left(u_{l+1}\right)^{p-1},
$$

where

$$
\hat{G}_{\hat{w}, i+\frac{1}{2}}=\kappa_{w} \frac{B\left(P_{w, i+\frac{1}{2}}\right) \hat{w}_{i}-B\left(P_{w, i+\frac{1}{2}}\right) \hat{w}_{i+1}}{h_{i+\frac{1}{2}}},
$$

with

$$
P_{w, i+\frac{1}{2}}=\frac{h_{i+\frac{1}{2}} x_{i+\frac{1}{2}}}{\kappa_{w}} \sigma
$$

and where

$$
\sigma=\hat{s} \frac{\hat{s}-s^{n}}{\Delta t}
$$

We supplement this scheme by the boundary conditions

$$
\hat{v}_{0}=r^{n+1} \text { and } \hat{u}_{0}=g^{n+1},
$$

with $r^{n+1}$ and $g^{n+1}$ defined by (10).

We notice two important facts. First, the assumption $(A 7)$ ensures that $\hat{v}_{0}$ and $\hat{u}_{0}$ satisfy

$$
0 \leq \hat{v}_{0} \leq r^{*} \text { and } \quad 0 \leq \hat{u}_{0} \leq g^{*}
$$

Furthermore, since

$$
0 \leq \frac{\hat{s}-s^{n}}{\Delta t} \leq \alpha\left(g^{*}\right)^{p}
$$

and using the boundary condition for $\hat{v}$ at $x=1$ and the hypothesis (9), we rewrite $\hat{v}_{l+1}$ as

$$
\hat{v}_{l+1}=\omega \hat{v}_{l},
$$


with $\omega$ a positive constant. Then, it suffices to study the two decoupled linear systems which can be written as

$$
\mathbb{M}_{\hat{u}} \hat{U}=b_{\hat{u}} \quad \text { and } \quad \mathbb{M}_{\hat{v}} \hat{V}=b_{\hat{v}},
$$

with $\hat{U}=\left(\hat{u}_{1}, \cdots, \hat{u}_{l+1}\right)^{t}, \hat{V}=\left(\hat{v}_{1}, \cdots, \hat{v}_{l}\right)^{t}, \mathbb{M}_{\hat{u}} \in \mathcal{M}_{l+1}(\mathbb{R}), \mathbb{M}_{\hat{v}} \in \mathcal{M}_{l}(\mathbb{R})$, $b_{\hat{u}} \in \mathbb{R}^{l+1}$ and $b_{\hat{v}} \in \mathbb{R}^{l}$. The matrices $\mathbb{M}_{\hat{u}}$ and $\mathbb{M}_{\hat{v}}$ are tridiagonal. Moreover, $\mathbb{M}_{\hat{u}}$ and $\mathbb{M}_{\hat{v}}$ are M-matrices and thus invertible and monotone, see [8]. Since, $b_{\hat{u}} \geq 0$ and $b_{\hat{v}} \geq 0$, we deduce thanks to the induction hypothesis that $\hat{U} \geq 0$, $\hat{V} \geq 0$ and by (23) we conclude that $\hat{v}_{l+1} \geq 0$.

Finally, following the proof of [8], we show that for all $i \in\{1, \cdots, l+1\}$ we have

$$
\hat{u}_{i} \leq g^{*} \text { and } \hat{v}_{i} \leq r^{*} .
$$

Thus, $T^{n}$ stabilizes the set $\mathbb{K}$ and then, thanks to the Brouwer's fixed-point theorem, $T^{n}$ has a fixed-point in $\mathbb{K}$, denoted by $\left(u^{n+1}, v^{n+1}\right)$. Eventually, we construct $s^{n+1}$ by

$$
s^{n+1}=s^{n}+\Delta t \alpha\left(u_{l+1}^{n+1}\right)^{p} .
$$

Hence, we deduce the existence of $\left(s^{n+1}, u^{n+1}, v^{n+1}\right)$ solution to $(S)$ such that $u^{n+1}, v^{n+1}$ and $s^{n+1}$ satisfy (19). As a by-product, we deduce (20) since $u^{n+1} \in$ $\mathbb{K}$ and $s^{n+1} \geq s^{n} \geq 0$.

\section{Discrete $L^{2}\left(0, T ; H^{1}(0,1)\right)$ estimate on the ap- proximate solutions}

Following $[2,3]$, we establish in this section a discrete $L^{2}\left(0, T ; H^{1}(0,1)\right)$ estimate. This estimate is the discrete counterpart of Lemma 3.4 in [2]: under the assumptions $(A 1)-(A 7)$ and if we assume that $g(t)=g^{*}$ and $r(t)=r^{*}$ on $[0,+\infty)$. Then, we have the following estimate

$$
\begin{gathered}
\int_{0}^{T} s^{0}\left[\frac{1}{\alpha^{1 / p}}\left(s^{\prime}\right)^{(p+1) / p}+\frac{1}{2 \alpha^{2 / p}}\left(s^{\prime}\right)^{(p+2) / p}\right] d t+\kappa_{u} \int_{0}^{T} \int_{0}^{1}\left|\partial_{x} u\right|^{2} d x d t \\
+\kappa_{v} \gamma \int_{0}^{T} \int_{0}^{1}\left|\partial_{x} v\right|^{2} d x d t \leq \frac{1}{2} \int_{0}^{T} \int_{0}^{1} s s^{\prime}\left(u^{2}+\gamma v^{2}\right) d x d t \\
+\frac{s^{2}(T)}{2}\left(\left(g^{*}\right)^{2}+g^{*}+\gamma\left(r^{*}\right)^{2}\right)+\frac{1}{2} \int_{0}^{1} s_{0}^{2}\left[\left(u_{0}\left(s_{0} x\right)-g^{*}\right)^{2}+\gamma\left(v_{0}\left(s_{0} x\right)-r^{*}\right)^{2}\right] d x .
\end{gathered}
$$

In order to state the discrete version of (24), let us remark that there exists a positive constant $\tau$ such that

$$
B^{c}(x) \geq \tau, \quad \forall x \in \mathbb{R}
$$

We refer to Appendix A where we prove the existence of $\tau$. Moreover, we notice that in the case of the upwind fluxes or the Scharfetter-Gummel fluxes $\tau=1$. We now state the discrete $L^{2}\left(0, T ; H^{1}(0,1)\right)$ estimate. 
Proposition 4.1. Let the hypothesis $(A 1)-(A 7)$ and $(9)$ hold and assume that $g(t)=g^{*}$ and $r(t)=r^{*}$ on $[0,+\infty)$. Then, for a given $\Delta t$ and a given mesh $\mathcal{T}$ we have

$$
\begin{aligned}
& \sum_{n=0}^{N_{T}-1} \Delta t s^{0}\left[\frac{1}{\alpha^{1 / p}}\left(\frac{s^{n+1}-s^{n}}{\Delta t}\right)^{(p+1) / p}+\frac{1}{2 \alpha^{2 / p}}\left(\frac{s^{n+1}-s^{n}}{\Delta t}\right)^{(p+2) / p}\right] \\
& +\kappa_{u} \tau \sum_{n=0}^{N_{T}-1} \Delta t \sum_{i=0}^{l} \frac{\left(u_{i+1}^{n+1}-u_{i}^{n+1}\right)^{2}}{h_{i+\frac{1}{2}}}+\gamma \kappa_{v} \tau \sum_{n=0}^{N_{T}-1} \Delta t \sum_{i=0}^{l} \frac{\left(v_{i+1}^{n+1}-v_{i}^{n+1}\right)^{2}}{h_{i+\frac{1}{2}}} \\
& \leq\left(s^{N_{T}}\right)^{2}\left(\left(g^{*}\right)^{2}+g^{*}+\gamma\left(r^{*}\right)^{2}\right)+\frac{\left(s_{0}\right)^{2}}{2} \sum_{i=1}^{l} h_{i}\left[\left(u_{i}^{0}-g^{*}\right)^{2}+\gamma\left(v_{i}^{0}-r^{*}\right)^{2}\right] \\
& +\frac{1}{2} \sum_{n=0}^{N_{T}-1} \Delta t \sum_{i=0}^{l} h_{i} \sigma^{n+1}\left[\left(u_{i}^{n+1}\right)^{2}+\gamma\left(v_{i}^{n+1}\right)^{2}\right] .
\end{aligned}
$$

\section{Proof of Proposition 4.1.}

We multiply (4) by $\Delta t\left(u_{i}^{n+1}-g^{*}\right)$ and we sum over $i$ and $n$, we obtain $E+F=G$, with

$$
\begin{aligned}
E & =\sum_{n=0}^{N_{T}-1} \Delta t \sum_{i=1}^{l} h_{i} s^{n+1} \frac{\left(s^{n+1} u_{i}^{n+1}-s^{n} u_{i}^{n}\right)}{\Delta t}\left(u_{i}^{n+1}-g^{*}\right) \\
F & =\sum_{n=0}^{N_{T}-1} \Delta t \sum_{i=1}^{l}\left(\mathcal{F}_{u, i+\frac{1}{2}}^{n+1}-\mathcal{F}_{u, i-\frac{1}{2}}^{n+1}\right)\left(u_{i}^{n+1}-g^{*}\right) \\
G & =\sum_{n=0}^{N_{T}-1} \Delta t \sum_{i=1}^{l} h_{i}\left(s^{n+1}\right)^{2} f\left(u_{i}^{n+1}, v_{i}^{n+1}\right)\left(u_{i}^{n+1}-g^{*}\right) .
\end{aligned}
$$

We notice that we can rewrite $E$ as

$$
\begin{aligned}
E=\sum_{n=0}^{N_{T}-1} \sum_{i=1}^{l} h_{i} s^{n+1}\left[s^{n+1}\left(u_{i}^{n+1}-g^{*}\right)-s^{n}\right. & \left(u_{i}^{n}-g^{*}\right) \\
& \left.+g^{*}\left(s^{n+1}-s^{n}\right)\right]\left(u_{i}^{n+1}-g^{*}\right) .
\end{aligned}
$$

Using the formula $(a-b) a \geq\left(a^{2}-b^{2}\right) / 2$ we obtain

$$
\begin{aligned}
E \geq \frac{1}{2} \sum_{n=0}^{N_{T}-1} \sum_{i=1}^{l} h_{i}\left[\left(s^{n+1}\right)^{2}\left(u_{i}^{n+1}-g^{*}\right)^{2}\right. & \left.-\left(s^{n}\right)^{2}\left(u_{i}^{n}-g^{*}\right)^{2}\right] \\
& +g^{*} \sum_{n=0}^{N_{T}-1} \Delta t \sum_{i=1}^{l} h_{i} \sigma^{n+1}\left(u_{i}^{n+1}-g^{*}\right) .
\end{aligned}
$$

Thanks to Theorem 2.1 we know that $u_{i}^{n+1} \geq 0$ for all $1 \leq i \leq l$ and $0 \leq n \leq$ 
$N_{T}-1$ which leads to

$$
\begin{aligned}
E \geq \frac{1}{2} \sum_{i=1}^{l} h_{i}\left[\left(s^{N_{T}}\right)^{2}\left(u_{i}^{N_{T}}-g^{*}\right)^{2}-\left(s^{0}\right)^{2}\left(u_{i}^{0}-g^{*}\right)^{2}\right] & \\
& -\left(g^{*}\right)^{2} \sum_{n=0}^{N_{T}-1} \Delta t \sum_{i=1}^{l} h_{i} \sigma^{n+1},
\end{aligned}
$$

and since $\sum_{i=1}^{l} h_{i}=1$ we have

$$
E \geq \frac{1}{2} \sum_{i=1}^{l} h_{i}\left[\left(s^{N_{T}}\right)^{2}\left(u_{i}^{N_{T}}-g^{*}\right)^{2}-\left(s^{0}\right)^{2}\left(u_{i}^{0}-g^{*}\right)^{2}\right]-\left(g^{*}\right)^{2} \sum_{n=0}^{N_{T}-1} \Delta t \sigma^{n+1} .
$$

Now, let us notice, by monotonicity of $\left(s^{n}\right)_{0 \leq n \leq N_{T}}$ thanks to (20), that for all $0 \leq n \leq N_{T}-1$

$$
\Delta t \sigma^{n+1}=\left(s^{n+1}\right)^{2}-s^{n+1} s^{n} \leq\left(s^{n+1}\right)^{2}-\left(s^{n}\right)^{2} .
$$

Then, we deduce that

$$
E \geq-\frac{1}{2} \sum_{i=1}^{l} h_{i}\left(s^{0}\right)^{2}\left(u_{i}^{0}-g^{*}\right)^{2}-\left(g^{*}\right)^{2} \sum_{n=0}^{N_{T}-1}\left(\left(s^{n+1}\right)^{2}-\left(s^{n}\right)^{2}\right) .
$$

Hence, we obtain

$$
E \geq-\frac{1}{2} \sum_{i=1}^{l} h_{i}\left(s^{0}\right)^{2}\left(u_{i}^{0}-g^{*}\right)^{2}-\left(g^{*}\right)^{2}\left(s^{N_{T}}\right)^{2}
$$

For $F$, a summation by parts leads to $F=F_{1}+F_{2}$ where $F_{1}$ contains the numerical fluxes and $F_{2}$ the boundary terms:

$$
\begin{aligned}
& F_{1}=-\sum_{n=0}^{N_{T}-1} \Delta t \sum_{i=0}^{l} \mathcal{F}_{u, i+\frac{1}{2}}^{n+1}\left(u_{i+1}^{n+1}-u_{i}^{n+1}\right), \\
& F_{2}=\sum_{n=0}^{N_{T}-1} \Delta t \mathcal{F}_{u, l+\frac{1}{2}}^{n+1}\left(u_{l+1}^{n+1}-g^{*}\right) .
\end{aligned}
$$

For $F_{1}$, we use the decomposition formula (17) and we get

$$
\begin{aligned}
F_{1}=\kappa_{u} \sum_{n=0}^{N_{T}-1} \Delta t \sum_{i=0}^{l} B^{c} & \left(P_{u, i+\frac{1}{2}}^{n+1}\right) \frac{\left(u_{i+1}^{n+1}-u_{i}^{n+1}\right)^{2}}{h_{i+\frac{1}{2}}} \\
& +\frac{1}{2} \sum_{n=0}^{N_{T}-1} \Delta t \sum_{i=0}^{l} x_{i+\frac{1}{2}} \sigma^{n+1}\left(\left(u_{i+1}^{n+1}\right)^{2}-\left(u_{i}^{n+1}\right)^{2}\right) .
\end{aligned}
$$

We reorder the convective terms and since by Lemma $7.1, B^{c}(x) \geq \tau$ for all $x \in \mathbb{R}$, we obtain

$$
\begin{aligned}
F_{1} \geq \kappa_{u} \tau \sum_{n=0}^{N_{T}-1} & \Delta t \sum_{i=0}^{l} \frac{\left(u_{i+1}^{n+1}-u_{i}^{n+1}\right)^{2}}{h_{i+\frac{1}{2}}} \\
& -\frac{1}{2} \sum_{n=0}^{N_{T}-1} \Delta t \sum_{i=0}^{l} h_{i} \sigma^{n+1}\left(u_{i}^{n+1}\right)^{2}+\frac{1}{2} \sum_{n=0}^{N_{T}-1} \Delta t \sigma^{n+1}\left(u_{l+1}^{n+1}\right)^{2} .
\end{aligned}
$$


Using equation (3) we have $\alpha\left(u_{l+1}^{n+1}\right)^{p}=\left(s^{n+1}-s^{n}\right) / \Delta t$ and the inequality $\sigma^{n+1} \geq s^{0}\left(s^{n+1}-s^{n}\right) / \Delta t$ for the last term of the right hand side, we deduce that

$$
\begin{aligned}
F_{1} \geq & \kappa_{u} \tau \sum_{n=0}^{N_{T}-1} \Delta t \sum_{i=0}^{l} \frac{\left(u_{i+1}^{n+1}-u_{i}^{n+1}\right)^{2}}{h_{i+\frac{1}{2}}} \\
& -\frac{1}{2} \sum_{n=0}^{N_{T}-1} \Delta t \sum_{i=0}^{l} h_{i} \sigma^{n+1}\left(u_{i}^{n+1}\right)^{2}+\sum_{n=0}^{N_{T}-1} \frac{\Delta t s^{0}}{2 \alpha^{2 / p}}\left(\frac{s^{n+1}-s^{n}}{\Delta t}\right)^{(p+2) / p} .
\end{aligned}
$$

For $F_{2}$, we apply (13) and we have

$$
F_{2}=\sum_{n=0}^{N_{T}-1} \Delta t \sigma^{n+1} u_{l+1}^{n+1}-g^{*} \sum_{n=0}^{N_{T}-1} \Delta t \sigma^{n+1}
$$

Using equation (3), as for the term $F_{1}$, we obtain

$$
F_{2}=\sum_{n=0}^{N_{T}-1} \Delta t \frac{s^{n+1}}{\alpha^{1 / p}}\left(\frac{s^{n+1}-s^{n}}{\Delta t}\right)^{(p+1) / p}-g^{*} \sum_{n=0}^{N_{T}-1} \Delta t \sigma^{n+1} .
$$

Applying the inequality (26) and the fact that $s^{n+1} \geq s^{0}$ for all $0 \leq n \leq N_{T}-1$ we infer that

$$
F_{2} \geq \sum_{n=0}^{N_{T}-1} \frac{\Delta t s^{0}}{\alpha^{1 / p}}\left(\frac{s^{n+1}-s^{n}}{\Delta t}\right)^{(p+1) / p}-g^{*}\left(s^{N_{T}}\right)^{2} .
$$

We conclude that

$$
\begin{gathered}
F \\
\geq \kappa_{u} \tau \sum_{n=0}^{N_{T}-1} \Delta t \sum_{i=0}^{l} \frac{\left(u_{i+1}^{n+1}-u_{i}^{n+1}\right)^{2}}{h_{i+\frac{1}{2}}}-\frac{1}{2} \sum_{n=0}^{N_{T}-1} \Delta t \sum_{i=0}^{l} h_{i} \sigma^{n+1}\left(u_{i}^{n+1}\right)^{2} \\
+\sum_{n=0}^{N_{T}-1} \frac{\Delta t s^{0}}{2 \alpha^{2 / p}}\left(\frac{s^{n+1}-s^{n}}{\Delta t}\right)^{(p+2) / p}+\sum_{n=0}^{N_{T}-1} \frac{\Delta t s^{0}}{\alpha^{1 / p}}\left(\frac{s^{n+1}-s^{n}}{\Delta t}\right)^{(p+1) / p} \\
-g^{*}\left(s^{N_{T}}\right)^{2}
\end{gathered}
$$

Then, we obtain from equation $E+F=G$, inequalities (27) and (28)

$$
\begin{aligned}
& \sum_{n=0}^{N_{T}-1} \Delta t s^{0}\left[\frac{1}{\alpha^{1 / p}}\left(\frac{s^{n+1}-s^{n}}{\Delta t}\right)^{(p+1) / p}+\frac{1}{2 \alpha^{2 / p}}\left(\frac{s^{n+1}-s^{n}}{\Delta t}\right)^{(p+2) / p}\right] \\
& +\kappa_{u} \tau \sum_{n=0}^{N_{T}-1} \Delta t \sum_{i=0}^{l} \frac{\left(u_{i+1}^{n+1}-u_{i}^{n+1}\right)^{2}}{h_{i+\frac{1}{2}}} \leq\left(s^{N_{T}}\right)^{2}\left(\left(g^{*}\right)^{2}+g^{*}\right)+\frac{\left(s_{0}\right)^{2}}{2} \sum_{i=1}^{l} h_{i}\left(u_{i}^{0}-g^{*}\right)^{2} \\
& +\sum_{n=0}^{N_{T}-1} \Delta t \sum_{i=1}^{l} h_{i}\left(s^{n+1}\right)^{2} f\left(u_{i}^{n+1}, v_{i}^{n+1}\right)\left(u_{i}^{n+1}-g^{*}\right)+\frac{1}{2} \sum_{n=0}^{N_{T}-1} \Delta t \sum_{i=0}^{l} h_{i} \sigma^{n+1}\left(u_{i}^{n+1}\right)^{2} .
\end{aligned}
$$

As previously, we multiply (5) by $\gamma \Delta t\left(v^{n+1}-r^{*}\right)$ and we sum over $i$ and $n$, so that we obtain similarly $E+F=G$. Thus, applying the same techniques as 
before, we deduce that

$$
\begin{aligned}
& \gamma \kappa_{v} \tau \sum_{n=0}^{N_{T}-1} \Delta t \sum_{i=0}^{l} \frac{\left(v_{i+1}^{n+1}-v_{i}^{n+1}\right)^{2}}{h_{i+\frac{1}{2}}} \leq\left(s^{N_{T}}\right)^{2} \gamma\left(r^{*}\right)^{2} \\
& +\frac{\gamma}{2} \sum_{n=0}^{N_{T}-1} \Delta t \sum_{i=0}^{l} h_{i} \sigma^{n+1}\left(v_{i}^{n+1}\right)^{2}+\frac{\gamma\left(s_{0}\right)^{2}}{2} \sum_{i=1}^{l} h_{i}\left(v_{i}^{0}-r^{*}\right)^{2} \\
& -\gamma \sum_{n=0}^{N_{T}-1} \Delta t \sum_{i=1}^{l} h_{i}\left(s^{n+1}\right)^{2} f\left(u_{i}^{n+1}, v_{i}^{n+1}\right)\left(v_{i}^{n+1}-r^{*}\right) .
\end{aligned}
$$

Finally, we notice that, for $0 \leq n \leq N_{T}-1$ and $1 \leq i \leq l$, we have

$$
\begin{aligned}
f\left(u_{i}^{n+1}, v_{i}^{n+1}\right)\left(u_{i}^{n+1}-g^{*}\right)-\gamma f\left(u_{i}^{n+1}, v_{i}^{n+1}\right)\left(v_{i}^{n+1}-r^{*}\right) \\
=-\beta\left(\gamma v_{i}^{n+1}-u_{i}^{n+1}\right)^{2} \leq 0 .
\end{aligned}
$$

Thus, we sum (29) and (30) and we deduce, thanks to (31), the inequality (25).

Corollary 4.1. Let the hypothesis $(A 1)-(A 7)$ and (9) hold and assume that $g(t)=g^{*}$ and $r(t)=r^{*}$ on $[0,+\infty)$. Then, for a given $\Delta t$ and a given mesh $\mathcal{T}$, there exists a constant $C$ which depends on $g^{*}, r^{*}, \tau,\left\|u^{0}-g^{*}\right\|_{0}^{2}$ and $\left\|v^{0}-r^{*}\right\|_{0}^{2}$ such that

$\kappa_{u} \sum_{n=0}^{N_{T}-1} \Delta t \sum_{i=0}^{l} \frac{\left(u_{i+1}^{n+1}-u_{i}^{n+1}\right)^{2}}{h_{i+\frac{1}{2}}}+\kappa_{v} \gamma \sum_{n=0}^{N_{T}-1} \Delta t \sum_{i=0}^{l} \frac{\left(v_{i+1}^{n+1}-v_{i}^{n+1}\right)^{2}}{h_{i+\frac{1}{2}}} \leq C\left(s^{N_{T}}\right)^{2}$.

Proof of Corollary 4.1.

Since $s_{0} \leq s^{N_{T}}$ we deduce from Proposition 4.1 that

$$
\begin{array}{r}
\kappa_{u} \tau \sum_{n=0}^{N_{T}-1} \Delta t \sum_{i=0}^{l} \frac{\left(u_{i+1}^{n+1}-u_{i}^{n+1}\right)^{2}}{h_{i+\frac{1}{2}}}+\gamma \kappa_{v} \tau \sum_{n=0}^{N_{T}-1} \Delta t \sum_{i=0}^{l} \frac{\left(v_{i+1}^{n+1}-v_{i}^{n+1}\right)^{2}}{h_{i+\frac{1}{2}}} \\
\leq\left(s^{N_{T}}\right)^{2}\left[\left(g^{*}\right)^{2}+g^{*}+\gamma\left(r^{*}\right)^{2}+\frac{1}{2} \sum_{i=1}^{l} h_{i}\left[\left(u_{i}^{0}-g^{*}\right)^{2}+\gamma\left(v_{i}^{0}-r^{*}\right)^{2}\right]\right. \\
\left.+\frac{\left(g^{*}\right)^{2}+\gamma\left(r^{*}\right)^{2}}{2}\right] .
\end{array}
$$

This concludes the proof of Corollary 4.1.

\section{The long time behavior of the approximate penetration depth}

In this section, we prove Theorem 2.2. As already mentioned, in the continuous setting the key idea is to establish an energy equality and then to deduce the $\sqrt{t}$-bounds for the penetration depth. This equality is established in Lemma 3.3 
in [2], see also Lemma 2.4 in [3], and could be stated as follows: assume that the assumptions $(A 1)-(A 7)$ hold, then we have

$$
\begin{aligned}
& \int_{0}^{1} s^{2}(T) x(u(x, T)+v(x, T)) d x+\frac{1}{2} s^{2}(T)+\kappa_{u} \int_{0}^{T} \int_{0}^{1} \partial_{x} u(x, t) d x d t \\
& +\kappa_{v} \int_{0}^{T} \int_{0}^{1} \partial_{x} v(x, t) d x d t=\frac{1}{2} s_{0}^{2}+\int_{0}^{1} s_{0}^{2} x\left(u_{0}\left(s_{0} x\right)+v_{0}\left(s_{0} x\right)\right) d x
\end{aligned}
$$

However, at the discrete level the techniques employed in $[2,3]$ for the establishment of (33) do not directly apply. Hence, in order to prove Theorem 2.2 we establish two discrete energy inequalities and then we will deduce the $\sqrt{t}$-bounds satisfied by the approximate penetration depth.

\subsection{The lower bound}

In this section, we show the lower bound of (21). To this end, we first state an energy inequality.

Proposition 5.1. Let the hypothesis (A1)-(A7) and (9) hold. Then, for a given $\Delta t$ and a given mesh $\mathcal{T}$ a solution to $(S)$ satisfies

$$
\begin{aligned}
\frac{3 g^{*}+3 r^{*}+1}{2}\left(s^{N_{T}}\right)^{2} & +\kappa_{u} \sum_{n=0}^{N_{T}-1} \Delta t \sum_{i=0}^{l} B\left(P_{u, i+\frac{1}{2}}^{n+1}\right)\left(u_{i+1}^{n+1}-u_{i}^{n+1}\right) \\
& +\kappa_{v} \sum_{n=0}^{N_{T}-1} \Delta t \sum_{i=0}^{l} B\left(P_{v, i+\frac{1}{2}}^{n+1}\right)\left(v_{i+1}^{n+1}-v_{i}^{n+1}\right) \geq 0 .
\end{aligned}
$$

\section{Proof of Proposition 5.1.}

We multiply (4) by $\Delta t x_{i}$ and we sum over $i$ and $n$, we obtain $E+F=G$, with

$$
\begin{aligned}
E & =\sum_{n=0}^{N_{T}-1} \Delta t \sum_{i=1}^{l} h_{i} x_{i} s^{n+1} \frac{\left(s^{n+1} u_{i}^{n+1}-s^{n} u_{i}^{n}\right)}{\Delta t}, \\
F & =\sum_{n=0}^{N_{T}-1} \Delta t \sum_{i=1}^{l} x_{i}\left(\mathcal{F}_{u, i+\frac{1}{2}}^{n+1}-\mathcal{F}_{u, i-\frac{1}{2}}^{n+1}\right) \\
G & =\sum_{n=0}^{N_{T}-1} \Delta t \sum_{i=1}^{l} h_{i} x_{i}\left(s^{n+1}\right)^{2} f\left(u_{i}^{n+1}, v_{i}^{n+1}\right) .
\end{aligned}
$$

For $E$ we notice that

$E=\sum_{n=0}^{N_{T}-1} \sum_{i=1}^{l} h_{i} x_{i}\left(\left(s^{n+1}\right)^{2} u_{i}^{n+1}-\left(s^{n}\right)^{2} u_{i}^{n}\right)-\sum_{n=0}^{N_{T}-1} \sum_{i=1}^{l} h_{i} x_{i} s^{n}\left(s^{n+1}-s^{n}\right) u_{i}^{n}$.

Thanks to Theorem 2.1 we know that for all $1 \leq i \leq l$ and $0 \leq n \leq N_{T}-1$ we have $s^{n}\left(s^{n+1}-s^{n}\right) u_{i}^{n} \geq 0$ and we get

$$
E \leq \sum_{n=0}^{N_{T}-1} \sum_{i=1}^{l} h_{i} x_{i}\left(\left(s^{n+1}\right)^{2} u_{i}^{n+1}-\left(s^{n}\right)^{2} u_{i}^{n}\right) .
$$


Then, using the telescopic sum and the inequality $\sum_{i=1}^{l} h_{i} x_{i} \leq \sum_{i=1}^{l} h_{i}=1$ we have

$$
E \leq \sum_{i=1}^{l} h_{i} x_{i}\left(s^{N_{T}}\right)^{2} u_{i}^{N_{T}}-\sum_{i=1}^{l} h_{i} x_{i}\left(s^{0}\right)^{2} u_{i}^{0} \leq g^{*}\left(s^{N_{T}}\right)^{2} .
$$

For $F$, a summation by parts leads to

$$
F=-\sum_{n=0}^{N_{T}-1} \Delta t \sum_{i=0}^{l} h_{i+\frac{1}{2}} \mathcal{F}_{u, i+\frac{1}{2}}^{n+1}+\sum_{n=0}^{N_{T}-1} \Delta t \mathcal{F}_{u, l+\frac{1}{2}}^{n+1}
$$

Then, using the decomposition formula (16) and the equation (13), we obtain

$$
\begin{aligned}
F=\kappa_{u} \sum_{n=0}^{N_{T}-1} \Delta t \sum_{i=0}^{l} B & \left(P_{u, i+\frac{1}{2}}^{n+1}\right)\left(u_{i+1}^{n+1}-u_{i}^{n+1}\right) \\
& +\sum_{n=0}^{N_{T}-1} \Delta t \sum_{i=0}^{l} h_{i+\frac{1}{2}} x_{i+\frac{1}{2}} \sigma^{n+1} u_{i+1}^{n+1}+\sum_{n=0}^{N_{T}-1} \Delta t \sigma^{n+1} .
\end{aligned}
$$

Since $u_{i}^{n+1} \leq g^{*}$ for all $1 \leq i \leq l+1$, we deduce thanks to the inequality $\sum_{i=0}^{l} h_{i+\frac{1}{2}} x_{i+\frac{1}{2}} \leq \sum_{i=0}^{l} h_{i+\frac{1}{2}}=1$ that

$$
F \leq \kappa_{u} \sum_{n=0}^{N_{T}-1} \Delta t \sum_{i=0}^{l} B\left(P_{u, i+\frac{1}{2}}^{n+1}\right)\left(u_{i+1}^{n+1}-u_{i}^{n+1}\right)+\left(g^{*}+1\right) \sum_{n=0}^{N_{T}-1} \Delta t \sigma^{n+1} .
$$

Moreover, the inequality (26) yields to

$$
\begin{aligned}
F \leq \kappa_{u} \sum_{n=0}^{N_{T}-1} \Delta t \sum_{i=0}^{l} B\left(P_{u, i+\frac{1}{2}}^{n+1}\right)\left(u_{i+1}^{n+1}-u_{i}^{n+1}\right) & \\
& +\frac{g^{*}+1}{2} \sum_{n=0}^{N_{T}-1}\left(\left(s^{n+1}\right)^{2}-\left(s^{n}\right)^{2}\right) .
\end{aligned}
$$

Then, applying (35) and (36) in the equation $E+F=G$ we get

$$
\frac{3 g^{*}+1}{2}\left(s^{N_{T}}\right)^{2}+\kappa_{u} \sum_{n=0}^{N_{T}-1} \Delta t \sum_{i=0}^{l} B\left(P_{u, i+\frac{1}{2}}^{n+1}\right)\left(u_{i+1}^{n+1}-u_{i}^{n+1}\right) \geq G .
$$

If we use the same techniques for $v$ we have

$$
\frac{3 r^{*}}{2}\left(s^{N_{T}}\right)^{2}+\kappa_{v} \sum_{n=0}^{N_{T}-1} \Delta t \sum_{i=0}^{l} B\left(P_{v, i+\frac{1}{2}}^{n+1}\right)\left(v_{i+1}^{n+1}-v_{i}^{n+1}\right) \geq-G .
$$

Finally, we sum (37) and (38) and we deduce (34).

Now, let us show the lower bound of (21). Thanks to Proposition 5.1 we obtain the inequality $E+F+G \geq 0$ with 


$$
\begin{aligned}
& E=\frac{3 g^{*}+3 r^{*}+1}{2}\left(s^{N_{T}}\right)^{2}, \\
& F=\kappa_{u} \sum_{n=0}^{N_{T}-1} \Delta t \sum_{i=0}^{l} B\left(P_{u, i+\frac{1}{2}}^{n+1}\right)\left(u_{i+1}^{n+1}-u_{i}^{n+1}\right), \\
& G=\kappa_{v} \sum_{n=0}^{N_{T}-1} \Delta t \sum_{i=0}^{l} B\left(P_{v, i+\frac{1}{2}}^{n+1}\right)\left(v_{i+1}^{n+1}-v_{i}^{n+1}\right) .
\end{aligned}
$$

We rewrite $F$ as $F=F_{1}+F_{2}$ with

$$
\begin{aligned}
& F_{1}=\kappa_{u} \sum_{n=0}^{N_{T}-1} \Delta t \sum_{i=0}^{l}\left(B\left(P_{u, i+\frac{1}{2}}^{n+1}\right)-1\right)\left(u_{i+1}^{n+1}-u_{i}^{n+1}\right), \\
& F_{2}=\kappa_{u} \sum_{n=0}^{N_{T}-1} \Delta t \sum_{i=0}^{l}\left(u_{i+1}^{n+1}-u_{i}^{n+1}\right) .
\end{aligned}
$$

For $\left|F_{1}\right|$, the Cauchy-Schwarz inequality yields to

$$
\begin{aligned}
\left|F_{1}\right| \leq \kappa_{u} \sum_{n=0}^{N_{T}-1} \Delta t\left(\sum_{i=0}^{l} h_{i+\frac{1}{2}}\left(B\left(P_{u, i+\frac{1}{2}}^{n+1}\right)-1\right)^{2}\right)^{1 / 2} & \left(\sum_{i=0}^{l} \frac{\left(u_{i+1}^{n+1}-u_{i}^{n+1}\right)^{2}}{h_{i+\frac{1}{2}}}\right)^{1 / 2} .
\end{aligned}
$$

Since $0 \leq B(x) \leq 1$ for $x \geq 0$ then $(B(x)-1)^{2} \leq 1$ for $x \geq 0$ and we obtain

$$
\left|F_{1}\right| \leq \kappa_{u} \sum_{n=0}^{N_{T}-1} \Delta t\left(\sum_{i=0}^{l} h_{i+\frac{1}{2}}\right)^{1 / 2}\left(\sum_{i=0}^{l} \frac{\left(u_{i+1}^{n+1}-u_{i}^{n+1}\right)^{2}}{h_{i+\frac{1}{2}}}\right)^{1 / 2} .
$$

Using the fact that $\sum_{i=0}^{l} h_{i+\frac{1}{2}}=1$ and the Cauchy-Schwarz inequality lead to

$$
\begin{aligned}
\left|F_{1}\right| & \leq \kappa_{u}\left(\sum_{n=0}^{N_{T}-1} \Delta t\right)^{1 / 2}\left(\sum_{n=0}^{N_{T}-1} \Delta t \sum_{i=0}^{l} \frac{\left(u_{i+1}^{n+1}-u_{i}^{n+1}\right)^{2}}{h_{i+\frac{1}{2}}}\right)^{1 / 2} \\
& \leq \sqrt{\kappa_{u} T}\left(\kappa_{u} \sum_{n=0}^{N_{T}-1} \Delta t \sum_{i=0}^{l} \frac{\left(u_{i+1}^{n+1}-u_{i}^{n+1}\right)^{2}}{h_{i+\frac{1}{2}}}\right)^{1 / 2} .
\end{aligned}
$$

Thanks to Corollary 4.1, we deduce the existence of a constant $c_{1}$ independent of $\Delta t$ such that

$$
\left|F_{1}\right| \leq c_{1} \sqrt{\kappa_{u} T} s^{N_{T}} .
$$

Then, we use the generalized Young inequality

$$
a b \leq \epsilon \frac{a^{r}}{r}+\frac{b^{q}}{q \epsilon^{q / r}}, \quad \text { with } \frac{1}{r}+\frac{1}{q}=1 \text { and } 1<r, q<\infty,
$$


with $a=c_{1} s^{N_{T}}, b=\sqrt{\kappa_{u} T}, r=2$ and $\epsilon=4 / g^{*}$ and we deduce the existence of a positive constant, still denoted $c_{1}$, independent of $\Delta t$ such that

$$
\left|F_{1}\right| \leq c_{1}\left(s^{N_{T}}\right)^{2}+\frac{\kappa_{u} g^{*}}{8} T .
$$

For $F_{2}$, using the telescopic sum and (3) we have

$$
F_{2}=\sum_{n=0}^{N_{T}-1} \frac{\Delta t \kappa_{u}}{\alpha^{1 / p}}\left(\frac{s^{n+1}-s^{n}}{\Delta t}\right)^{1 / p}-\kappa_{u} g^{*} T .
$$

In the case where $p>1$, applying Hölder inequality with exponents $p$ and $p /(p-1)$, we get

$$
\begin{aligned}
\sum_{n=0}^{N_{T}-1} \frac{\Delta t \kappa_{u}}{\alpha^{1 / p}}\left(\frac{s^{n+1}-s^{n}}{\Delta t}\right)^{1 / p} & \leq \frac{\kappa_{u}}{\alpha^{1 / p}}\left(\sum_{n=0}^{N_{T}-1} \Delta t\left(\frac{s^{n+1}-s^{n}}{\Delta t}\right)\right)^{1 / p} T^{(p-1) / p} \\
& \leq \frac{\kappa_{u}}{\alpha^{1 / p}}\left(s^{N_{T}}\right)^{1 / p} T^{(p-1) / p} .
\end{aligned}
$$

Then, we apply the generalized Young inequality (39) with $a=\left(\kappa_{u} s^{N_{T}} / \alpha\right)^{1 / p}$, $b=\left(\kappa_{u} T\right)^{(p-1) / p}, r=p$ and $\epsilon=\left(4(p-1) / g^{*} p\right)^{p-1}$ and we deduce the existence of a constant $c_{2}$ independent of $\Delta t$ such that

$$
\sum_{n=0}^{N_{T}-1} \frac{\Delta t \kappa_{u}}{\alpha^{1 / p}}\left(\frac{s^{n+1}-s^{n}}{\Delta t}\right)^{1 / p} \leq c_{2} s^{N_{T}}+\frac{\kappa_{u} g^{*}}{4} T .
$$

In the case where $p=1$ using the telescopic sum we obtain

$$
\sum_{n=0}^{N_{T}-1} \frac{\Delta t \kappa_{u}}{\alpha}\left(\frac{s^{n+1}-s^{n}}{\Delta t}\right) \leq \frac{\kappa_{u}}{\alpha} s^{N_{T}} \leq \frac{\kappa_{u}}{\alpha} s^{N_{T}}+\frac{\kappa_{u} g^{*}}{4} T .
$$

Thus, in both cases, we deduce the existence of a constant, still denoted $c_{2}$, independent of $\Delta t$ such that

$$
F_{2} \leq c_{2} s^{N_{T}}-\frac{3 \kappa_{u} g^{*}}{4} T .
$$

For $G$, thanks to Corollary 4.1 we deduce that there exists a positive constant $c_{3}$ independent of $\Delta t$ such that

$$
\kappa_{v} \sum_{n=0}^{N_{T}-1} \Delta t \sum_{i=0}^{l} \frac{\left(v_{i+1}^{n+1}-v_{i}^{n+1}\right)^{2}}{h_{i+\frac{1}{2}}} \leq c_{3}\left(s^{N_{T}}\right)^{2} .
$$

Applying the Cauchy-Schwarz inequality, we obtain

$$
G \leq \kappa_{v} \sum_{n=0}^{N_{T}-1} \Delta t\left(\sum_{i=0}^{l} h_{i+\frac{1}{2}} B^{2}\left(P_{v, i+\frac{1}{2}}^{n+1}\right)\right)^{1 / 2}\left(\sum_{i=0}^{l} \frac{\left(v_{i+1}^{n+1}-v_{i}^{n+1}\right)^{2}}{h_{i+\frac{1}{2}}}\right)^{1 / 2} .
$$

Since $B(x) \leq 1$ for all $x \in \mathbb{R}^{+}$and $\sum_{i=0}^{l} h_{i+\frac{1}{2}}=1$ we deduce that

$$
G \leq \kappa_{v} \sum_{n=0}^{N_{T}-1} \Delta t\left(\sum_{i=0}^{l} \frac{\left(v_{i+1}^{n+1}-v_{i}^{n+1}\right)^{2}}{h_{i+\frac{1}{2}}}\right)^{1 / 2} .
$$


Using the Cauchy-Schwarz inequality we end up with

$$
G \leq \sqrt{\kappa_{v}}\left(\kappa_{v} \sum_{n=0}^{N_{T}-1} \Delta t \sum_{i=0}^{l} \frac{\left(v_{i+1}^{n+1}-v_{i}^{n+1}\right)^{2}}{h_{i+\frac{1}{2}}}\right)^{1 / 2} T^{1 / 2} .
$$

Hence, applying (42) we deduce the existence of a constant $c_{4}$ independent of $\Delta t$ such that

$$
G \leq c_{4} \sqrt{\kappa_{v} T} s^{N_{T}} .
$$

Thanks to the generalized Young inequality (39) with $a=c_{4} s^{N_{T}}, b=\sqrt{\kappa_{v} T}$, $r=2$ and $\epsilon=4 \kappa_{v} / \kappa_{u} g^{*}$ we deduce the existence of a constant, still denoted $c_{4}$, independent of $\Delta t$ such that

$$
G \leq c_{4}\left(s^{N_{T}}\right)^{2}+\frac{\kappa_{u} g^{*}}{8} T .
$$

Then, from equation $E+F+G \geq 0$ and (40)-(43) we deduce that

$$
\left(\frac{3 g^{*}+3 r^{*}+1}{2}+c_{1}+c_{4}\right)\left(s^{N_{T}}\right)^{2}+c_{2} s^{N_{T}} \geq \frac{\kappa_{u} g^{*}}{2} T .
$$

If $T>1$, again applying the generalized Young inequality (39) with $a=c_{2} s^{N_{T}}$, $b=T^{1 / 2}, r=2$ and $\epsilon=2 / \kappa_{u} g^{*}$ we obtain

$$
c_{2} s^{N_{T}} \leq c_{2} s^{N_{T}} T^{1 / 2} \leq \frac{c_{2}^{2}}{\kappa_{u} g^{*}}\left(s^{N_{T}}\right)^{2}+\frac{\kappa_{u} g^{*}}{4} T .
$$

We deduce the existence of a positive constant $c$, independent of $\Delta t$, such that

$$
s^{N_{T}} \geq c \sqrt{T} .
$$

In the case $0 \leq T \leq 1$, we have

$$
s^{N_{T}} \geq s_{0} \geq s_{0} T^{1 / 2} .
$$

This concludes the proof of the lower bound of (21).

\subsection{The upper bound}

In this section, we prove the upper bound of (21). As previously, we first establish an energy inequality:

Proposition 5.2. Let the hypothesis $(A 1)-(A 7)$ and (9) hold and assume that $g^{*}+r^{*}<1$. Then, for a given $\Delta t$ and a given mesh $\mathcal{T}$ a solution to $(S)$ satisfies

$$
\begin{aligned}
& \frac{1-\left(g^{*}+r^{*}\right)}{2}\left(s^{N_{T}}\right)^{2}+\kappa_{u} \sum_{n=0}^{N_{T}-1} \Delta t \sum_{i=0}^{l} B\left(P_{u, i+\frac{1}{2}}^{n+1}\right)\left(u_{i+1}^{n+1}-u_{i}^{n+1}\right) \\
&+\kappa_{v} \sum_{n=0}^{N_{T}-1} \Delta t \sum_{i=0}^{l} B\left(P_{v, i+\frac{1}{2}}^{n+1}\right)\left(v_{i+1}^{n+1}-v_{i}^{n+1}\right) \leq \sum_{i=1}^{l} h_{i} x_{i}\left(s_{0}\right)^{2}\left(u_{i}^{0}+v_{i}^{0}\right) \\
&+\frac{1-\left(g^{*}+r^{*}\right)}{2}\left(s^{0}\right)^{2} .
\end{aligned}
$$




\section{Proof of Proposition 5.2.}

We multiply (4) by $\Delta t x_{i}$ and we sum over $i$ and $n$, we obtain $E+F=G$, with

$$
\begin{aligned}
E & =\sum_{n=0}^{N_{T}-1} \Delta t \sum_{i=1}^{l} h_{i} x_{i} s^{n+1} \frac{\left(s^{n+1} u_{i}^{n+1}-s^{n} u_{i}^{n}\right)}{\Delta t}, \\
F & =\sum_{n=0}^{N_{T}-1} \Delta t \sum_{i=1}^{l} x_{i}\left(\mathcal{F}_{u, i+\frac{1}{2}}^{n+1}-\mathcal{F}_{u, i-\frac{1}{2}}^{n+1}\right) \\
G & =\sum_{n=0}^{N_{T}-1} \Delta t \sum_{i=1}^{l} h_{i} x_{i}\left(s^{n+1}\right)^{2} f\left(u_{i}^{n+1}, v_{i}^{n+1}\right) .
\end{aligned}
$$

For $E$ we notice that

$E=\sum_{n=0}^{N_{T}-1} \sum_{i=1}^{l} h_{i} x_{i}\left(\left(s^{n+1}\right)^{2} u_{i}^{n+1}-\left(s^{n}\right)^{2} u_{i}^{n}\right)-\sum_{n=0}^{N_{T}-1} \sum_{i=1}^{l} h_{i} x_{i} s^{n}\left(s^{n+1}-s^{n}\right) u_{i}^{n}$.

Then, using the telescopic sum and the fact that $s^{n}\left(s^{n+1}-s^{n}\right) u_{i}^{n} \leq \Delta t \sigma^{n+1} g^{*}$ for all $1 \leq i \leq l$ and $0 \leq n \leq N_{T}-1$ and $\sum_{i=1}^{l} h_{i} x_{i} \leq \sum_{i=1}^{l} h_{i}=1$ we have

$$
E \geq-\sum_{i=1}^{l} h_{i} x_{i}\left(s^{0}\right)^{2} u_{i}^{0}-g^{*} \sum_{n=0}^{N_{T}-1} \Delta t \sigma^{n+1}
$$

For $F$, a summation by parts leads to

$$
F=-\sum_{n=0}^{N_{T}-1} \Delta t \sum_{i=0}^{l} h_{i+\frac{1}{2}} \mathcal{F}_{u, i+\frac{1}{2}}^{n+1}+\sum_{n=0}^{N_{T}-1} \Delta t \mathcal{F}_{u, l+\frac{1}{2}}^{n+1}
$$

Then, using the decomposition formula (16) and the equation (13), we obtain

$$
\begin{aligned}
F=\kappa_{u} \sum_{n=0}^{N_{T}-1} \Delta t \sum_{i=0}^{l} B & \left(P_{u, i+\frac{1}{2}}^{n+1}\right)\left(u_{i+1}^{n+1}-u_{i}^{n+1}\right) \\
& +\sum_{n=0}^{N_{T}-1} \Delta t \sum_{i=0}^{l} h_{i+\frac{1}{2}} x_{i+\frac{1}{2}} \sigma^{n+1} u_{i+1}^{n+1}+\sum_{n=0}^{N_{T}-1} \Delta t \sigma^{n+1} .
\end{aligned}
$$

Since $\sigma^{n+1} u_{i}^{n+1} \geq 0$ for all $1 \leq i \leq l+1$ and $0 \leq n \leq N_{T}-1$, we deduce that

$$
F \geq \kappa_{u} \sum_{n=0}^{N_{T}-1} \Delta t \sum_{i=0}^{l} B\left(P_{u, i+\frac{1}{2}}^{n+1}\right)\left(u_{i+1}^{n+1}-u_{i}^{n+1}\right)+\sum_{n=0}^{N_{T}-1} \Delta t \sigma^{n+1} .
$$

Then, applying (45) and (46) in the equation $E+F=G$ we get

$$
\begin{array}{r}
\left(1-g^{*}\right) \sum_{n=1}^{N_{T}-1} \Delta t \sigma^{n+1}+\kappa_{u} \sum_{n=0}^{N_{T}-1} \Delta t \sum_{i=0}^{l} B\left(P_{u, i+\frac{1}{2}}^{n+1}\right)\left(u_{i+1}^{n+1}-u_{i}^{n+1}\right) \\
\leq G+\sum_{i=1}^{l} h_{i} x_{i}\left(s_{0}\right)^{2} u_{i}^{0} .
\end{array}
$$


If we use the same techniques for $v$ we have

$$
\begin{array}{r}
-r^{*} \sum_{n=1}^{N_{T}-1} \Delta t \sigma^{n+1}+\kappa \sum_{n=0}^{N_{T}-1} \Delta t \sum_{i=0}^{l} B\left(P_{v, i+\frac{1}{2}}^{n+1}\right)\left(v_{i+1}^{n+1}-v_{i}^{n+1}\right) \\
\leq-G+\sum_{i=1}^{l} h_{i} x_{i}\left(s_{0}\right)^{2} v_{i}^{0}
\end{array}
$$

Finally, summing (47) and (48) and using the hypothesis $g^{*}+r^{*}<1$ we deduce (44).

Now, let us establish the upper bound of (21). Thanks to Proposition 5.2 we obtain the inequality $E \leq F+G+H$ with

$$
\begin{aligned}
& E=\frac{1-\left(g^{*}+r^{*}\right)}{2}\left(s^{N_{T}}\right)^{2}, \\
& F=-\kappa_{u} \sum_{n=0}^{N_{T}-1} \Delta t \sum_{i=0}^{l} B\left(P_{u, i+\frac{1}{2}}^{n+1}\right)\left(u_{i+1}^{n+1}-u_{i}^{n+1}\right), \\
& G=-\kappa_{v} \sum_{n=0}^{N_{T}-1} \Delta t \sum_{i=0}^{l} B\left(P_{v, i+\frac{1}{2}}^{n+1}\right)\left(v_{i+1}^{n+1}-v_{i}^{n+1}\right), \\
& H=\sum_{i=1}^{l} h_{i} x_{i}\left(s_{0}\right)^{2}\left(u_{i}^{0}+v_{i}^{0}\right)+\frac{1-\left(g^{*}+r^{*}\right)}{2}\left(s^{0}\right)^{2} .
\end{aligned}
$$

We rewrite $F$ as $F=F_{1}+F_{2}$ with

$$
\begin{aligned}
& F_{1}=-\kappa_{u} \sum_{n=0}^{N_{T}-1} \Delta t \sum_{i=0}^{l}\left(B\left(P_{u, i+\frac{1}{2}}^{n+1}\right)-1\right)\left(u_{i+1}^{n+1}-u_{i}^{n+1}\right), \\
& F_{2}=-\kappa_{u} \sum_{n=0}^{N_{T}-1} \Delta t \sum_{i=0}^{l}\left(u_{i+1}^{n+1}-u_{i}^{n+1}\right)=-\kappa_{u} \sum_{n=0}^{N_{T}-1} \Delta t\left(u_{l+1}^{n+1}-u_{0}^{n+1}\right) .
\end{aligned}
$$

For $\left|F_{1}\right|$, using the same techniques as in the previous section, we obtain the existence of a positive constant $C_{1}$ independent of $\Delta t$ such that

$$
\left|F_{1}\right| \leq C_{1} \sqrt{\kappa_{u} T} s^{N_{T}} .
$$

Then, the generalized Young inequality (39) with $a=s^{N_{T}}, b=C_{1} \sqrt{\kappa_{u} T}, r=2$ and $\epsilon=\left(1-\left(g^{*}+r^{*}\right)\right) / 4$ leads to the existence of a positive constant, still denoted $C_{1}$, independent of $\Delta t$ such that

$$
\left|F_{1}\right| \leq \frac{1-\left(g^{*}+r^{*}\right)}{8}\left(s^{N_{T}}\right)^{2}+\frac{2 C_{1} \kappa_{u}}{1-\left(g^{*}+r^{*}\right)} T .
$$

For $F_{2}$, applying $u_{l+1}^{n+1} \geq 0$, we end up with

$$
F_{2} \leq \kappa_{u} g^{*} T
$$


We also rewrite $G$ as $G=G_{1}+G_{2}$ with

$$
\begin{aligned}
& G_{1}=-\kappa_{v} \sum_{n=0}^{N_{T}-1} \Delta t \sum_{i=0}^{l}\left(B\left(P_{v, i+\frac{1}{2}}^{n+1}\right)-1\right)\left(v_{i+1}^{n+1}-v_{i}^{n+1}\right), \\
& G_{2}=-\kappa_{v} \sum_{n=0}^{N_{T}-1} \Delta t \sum_{i=0}^{l}\left(v_{i+1}^{n+1}-v_{i}^{n+1}\right) .
\end{aligned}
$$

Then, using the same techniques as before for $\left|G_{1}\right|$ and $G_{2}$ we obtain

$$
\left|G_{1}\right| \leq \frac{1-\left(g^{*}+r^{*}\right)}{8}\left(s^{N_{T}}\right)^{2}+\frac{2 C_{2} \kappa_{v}}{1-\left(g^{*}+r^{*}\right)} T
$$

and

$$
G_{2} \leq \kappa_{v} r^{*} T
$$

with $C_{2}$ a constant independent of $\Delta t$. We apply (49)-(52) in the inequality $E \leq F+G+H$ and we end up with

$$
\frac{1-\left(g^{*}+r^{*}\right)}{4}\left(s^{N_{T}}\right)^{2} \leq\left[\kappa_{u} g^{*}+\kappa_{v} r^{*}+\frac{4 C_{3}}{1-\left(g^{*}+r^{*}\right)}\right] T+H,
$$

with $C_{3}=\max \left\{\kappa_{u} C_{1}, \kappa_{v} C_{2}\right\}$. Eventually, we deduce the existence of a positive constant $C$ independent of $\Delta t$ such that

$$
s^{N_{T}} \leq C \sqrt{1+T} .
$$

This concludes the proof of Theorem 2.2.

\section{$6 \quad$ Numerical experiments}

In this section, we present some numerical experiments. The test case is described in Table 1. Furthermore, as already observed in [8], the upwind scheme

\begin{tabular}{|c|c|c|c|c|}
\hline$\kappa_{u}$ & $\kappa_{v}$ & $s_{0}$ & $\alpha$ & $\beta$ \\
\hline 1 & 0.1 & 0.5 & 1 & 7.5 \\
\hline
\end{tabular}

Table 1: Definition of parameters used in the test case.

gives the same results than the Scharfetter-Gummel scheme. Since the ScharfetterGummel scheme is more accurate for diffusion-convection problem, we consider in the sequel that $B=B_{s g}$. Let us mention that the numerical results are obtained using Newton's method with a tolerance equal to $1 e-8$ on a uniform mesh made of 100 cells and $\Delta t=1 e-2$.

In Figure 1, we illustrate the behavior of $s$ in logarithmic scale for different values of $p$ up to $T=1000$ with $g^{*}=0.5, r^{*}=0.25, \gamma=2$ and $u_{0}(x)=v_{0}(x)=0.25$ for all $x \in[0,1]$. We observe that the penetration depth follows a $\sqrt{t}$-law of propagation. 

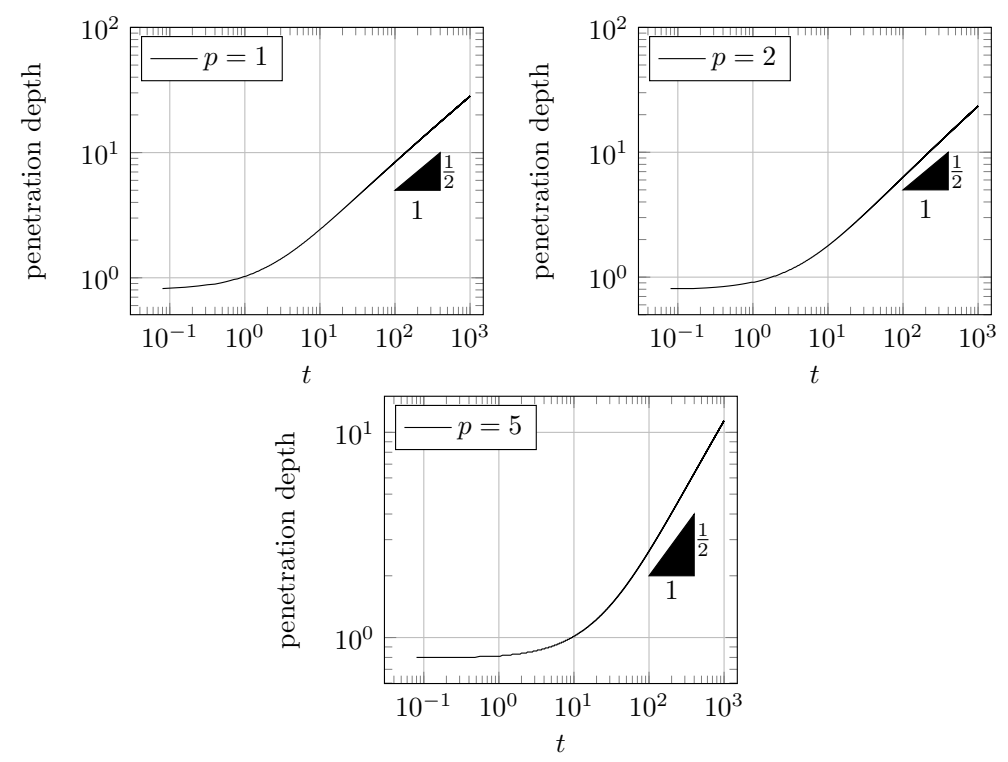

Figure 1: Profiles of $s$ in logarithmic scale with the Scharfetter-Gummel fluxes for different values of $p$ with $g^{*}+r^{*}<1$.

In Figure 2, we illustrate the behavior of $s$ in logarithmic scale for $p=2$ up to $T=1000$ with $g^{*}=15, r^{*}=2.25, \gamma=6.67$ and $u_{0}(x)=v_{0}(x)=1$ for all $x \in[0,1]$. We observe that, even if $g^{*}+r^{*}>1$, the scheme $(S)$ preserves the $\sqrt{t}$-law of propagation for the approximate penetration depth. Let us mention that we obtain similar profiles for $s$ for different values of $p$ in the case $g^{*}+r^{*}>1$.

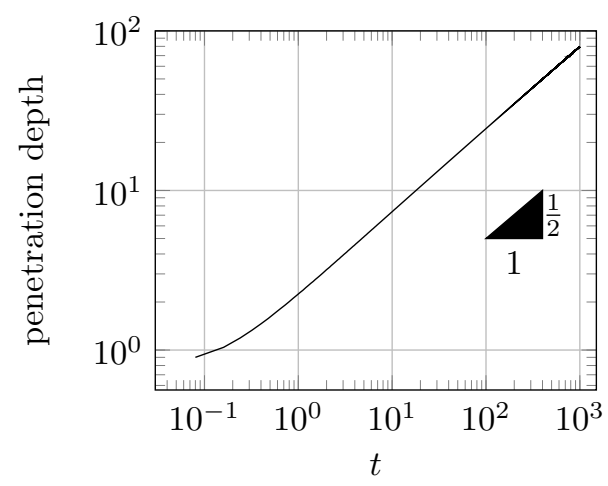

Figure 2: Profiles of $s$ in logarithmic scale with the Scharfetter-Gummel fluxes for $p=2$ with $g^{*}+r^{*}>1$. 


\section{Conclusions}

In this paper, we have justified the long time behavior of solutions of a finite volume scheme for the carbonation model introduced in [1]. Let us mention that the Corollary 4.1 and the techniques used in [8] could be adapted in order to show the convergence of $(S)$. Moreover, the numerical experiments seem to confirm the existence of a constant $\Lambda^{*}$ independent of $p$ such that $s(t) \sim \Lambda^{*} \sqrt{t}$ for $t$ large enough. Nevertheless, a rigorous justification of the existence of $\Lambda^{*}$ is still an open problem.

\section{Appendix A. Property of the $B$ function}

We prove in this Appendix the following result:

Lemma 7.1. Under the hypothesis (9), there exists a positive constant $\tau$ such that

$$
B^{c}(x)=\frac{B(x)+B(-x)}{2} \geq \tau, \quad \forall x \in \mathbb{R} .
$$

Proof of Lemma 7.1.

To this end, we first notice that thanks to $(9 \mathrm{~b})$ and $(9 \mathrm{~d})$ we have

$$
B(x) \geq-x .
$$

Then, $\lim _{x \rightarrow-\infty} B(x)=+\infty$. Moreover, we have

$$
B^{c}(x) \geq \frac{B(x)}{2} \quad \text { and } \quad B^{c}(x) \geq \frac{B(-x)}{2} .
$$

Thus, we deduce that

$$
\lim _{x \rightarrow-\infty} B^{c}(x)=\lim _{x \rightarrow+\infty} B^{c}(x)=+\infty .
$$

Finally, since $B(x)>0$ for $x \in \mathbb{R}$ we conclude that there exists $\tau>0$ such that (53) is satisfied.

\section{Acknowledgments}

The author is partially supported by the INRIA team RAPSODI, the ANR MOONRISE and the Labex CEMPI (ANR-11-LABX-0007-01). 


\section{Bibliography}

[1] T. Aiki and A. Muntean. Existence and uniqueness of solutions to a mathematical model predicting service life of concrete structure. Adv. Math. Sci. Appl., 19:109-129, 2009.

[2] T. Aiki and A. Muntean. Large time behavior of solutions to a movinginterface problem modeling concrete carbonation. Comm. Pure Appl. Anal., 9:1117-1129, 2010.

[3] T. Aiki and A. Muntean. A free-boundary problem for concrete carbonation: Front nucleation and rigorous justification of the $\sqrt{T}$-law of propagation. Interfaces and Free Bound., 15:167-180, 2013.

[4] T. Aiki and A. Muntean. Large-time asymptotics of moving-reaction interfaces involving nonlinear Henry's law and time-dependent Dirichlet data. Nonlinear Anal., 93:3-14, 2013.

[5] M. Bessemoulin-Chatard, C. Chainais-Hillairet, and M.-H. Vignal. Study of a finite volume scheme for the drift-diffusion system. asymptotic behavior in the quasi-neutral limit. SIAM J. Numer. Anal., 52:1666-1691, 2014.

[6] M. Böhm and A. Muntean. A moving-boundary problem for concrete carbonation: global existence and uniqueness of solutions. J. Math. Anal. Appl., 350:234-251, 2009.

[7] C. Chainais-Hillairet and J. Droniou. Finite volume schemes for noncoercive elliptic problems with neumann boundary conditions. IMA J. Numer. Anal., 31(1):61-85, 2011.

[8] C. Chainais-Hillairet, B. Merlet, and A. Zurek. Convergence of a finite volume scheme for a parabolic system with a free boundary modeling concrete carbonation. ESAIM: M2AN, https://doi.org/10.1051/m2an/2018002, 2018.

[9] M. Fila and P. Souplet. Existence of global solutions with slow decay and unbounded free boundary for a superlinear Stefan problem. Interfaces and Free Bound., 3:337-344, 2001.

[10] H. Ghidouche, P. Souplet, and D. Tarzia. Decay of global solutions, stability and blow-up for a reaction-diffusion problem with free boundary. Proc. Amer. Math. Soc., 129:781-792, 2001. 
[11] D. Hilhorst, F. Issard-Roch, and J.M. Roquejoffre. Large time behavior of the solutions to a one-dimensional Stefan problem with a kinetic condition at the free boundary. Euro. Jnl of Applied Mathematics, 15:297-313, 2004.

[12] A.M. Il'in. A difference scheme for a differential equation with a small parameter multiplying the highest derivative. Mat. Zametki, 6:237-248, 1969.

[13] D.L. Scharfetter and H.K. Gummel. Large signal analysis of a silicon read diode oscillator. IEEE Trans. Electron Dev., 16:64-77, 1969.

[14] D.A. Tarzia. Explicit and Approximated Solutions for Heat and Mass Transfer Problems with a Moving Interface, Advanced Topics in Mass Transfer, chapter 20. InTech, 2011. 